\title{
Framework of mechanical symmetry breaking theory and its application to innovative design
}

\author{
Qing-ying QIU, Shao-jian WANG, Pei-en FENG, Yu-xuan QI ${ }^{\dagger \dagger}$, Li-xin LI \\ (State Key Lab of CAD\&CG, Zhejiang University, Hangzhou 310027, China) \\ †E-mail: qiyvxuan@163.com
}

Received July 9, 2015; Revision accepted May 13, 2016; Crosschecked Oct. 10, 2016

\begin{abstract}
Symmetry breaking phenomena exist in many mechanical products. Based on an analysis of the variation in physical features in several design examples, mechanical symmetry breaking concept systems that consist of general requirements, working principles, and cross-scale mechanical structures are established from the macro to micro levels and in both machine assembly and the design of parts. The connection between symmetry breaking and product requirements is systematically studied. Eight design principles using symmetry breaking to achieve mechanical functions, performance, and constraints are proposed. The method and process of applying symmetry breaking in mechanical concept design are described. These principles, method, and process are then used in the concept design of a gear chain. With symmetry breaking characters, the transmission stability of the newly innovated gear chain is greatly improved, compared with traditional ones.
\end{abstract}

Key words: Mechanical symmetry breaking, Concept system, Design principle, Design method and process, Innovative instance

http://dx.doi.org/10.1631/jzus.A1500199

CLC number: TH112

\section{Introduction}

The symmetry breaking phenomenon plays an important role in many disciplines, and has drawn the attention of many scholars.

In biology, the example of the skull of flatfish was used to reveal the symmetry breaking phenomena of biological organs in the process of evolution caused, in this case, by the asymmetry of the ocean floor environment (Friedman, 2008). Saxena (2015) pointed out that symmetry breaking in the structure of any biological system is caused by either natural or man-controlled factors. Natural symmetry breaking reflects the complexities of biological systems,

\footnotetext{
Corresponding author

* Project supported by the National Natural Science Foundation of China (No. 51175455)

(i) ORCID: Qing-ying QIU, http://orcid.org/0000-0001-6884-3125; Yuxuan QI, http://orcid.org/0000-0002-7556-616X

(C) Zhejiang University and Springer-Verlag Berlin Heidelberg 2016
}

their organizational adaptations, and their mechanisms at cellular levels, while the controlled symmetry breaking of cells focuses on cell migration, morphogenesis, self-organization, and soluble and insoluble cues.

In the field of material structures, Xia and Xia (2012), for the first time, directed the growth of nanocrystal seeds and broke the symmetry of a face center cubic (FCC) lattice by incorporating a series of new shapes. Thus, nanocrystals with highly unsymmetrical shapes were obtained. Dong et al. (2012) obtained a transparency window within the absorptive dipole resonant regime by slightly reducing the translational symmetric arrangement of a dipole-like bar grating covered by a wave-guiding layer.

In the 3D image detecting and data processing fields, Angelo and Stefano (2013) proposed a new method capable of identifying the symmetry plane of human face. Their method is quite insensitive to local asymmetries, and is also repeatable and slightly conditioned by the acquisition process. 
In the field of engineering design and manufacture, Várkonyi (2006) pointed out that, in most cases, a small perturbation of the symmetry design of architectural forms and structures will yield an imperfect, i.e., worsened, structural configuration. However, in some cases, the imperfect symmetry design of architectural forms and structures emerges as an optimal design compared with their symmetric counterparts. Ge et al. (2011) found that the vibration of bridge structures is induced by symmetry breaking, which often leads to accidents. The TRIZ created by Altshuller proposed that replacing a symmetrical form with an asymmetrical form of the object can eliminate conflicts in the system (Silverstein et al., 2007). Suh (2004) proposed a minimum information axiom in axiomatic design theory, and claimed, by using symmetry structure as much as possible, to minimize the information need and reduce the design parameters. Despite Suh (2004)'s and Altshuller's efforts, the concept of symmetry breaking is not even mentioned (Hou and Xu, 2015). By analyzing a planetary gear train (PGT)'s graph and Hamming matrix representation, Rajasri et al. (2014) found that greater symmetry of a PGT results in more structural arrangements. However, they did not mention the necessity of the existence of symmetry breaking, i.e., asymmetrical structures within PGT. Masoumi et al. (2015) made a complete analysis of the dynamics of planetary gears and verified that the chaotic response indicates symmetry breaking in the dynamic systems. In the design for assembly (DFA) field, Boothroyd (2009) analyzed the effect of the symmetry of parts on their assembly efficiency, and two design rules were proposed: (1) try to design parts as symmetrical structures to save orienting manipulation; (2) increase asymmetry features of parts to assist orienting manipulation when parts cannot be designed as symmetrical structures.

Although there are a number of design cases of symmetry breaking structures, few references in the mechanical design field have systematically studied or proposed the concept and classification of mechanical symmetry breaking, not to mention the extraction and summarizing of working principles of symmetry breaking in mechanical engineering and the laws of their application in design.

Based on the analysis of more than 1000 design instances, the concept of mechanical symmetry breaking is established in this study. A series of con- cepts, including the space-time symmetry breaking concept of general requirements, the space-time symmetry breaking concept of working principles and the symmetry breaking concept of structures, are proposed. Rules for using symmetry breaking to satisfy product requirements are then extracted. Finally, the method and processes of applying symmetry breaking are proposed, and an instance of product innovation using symmetry breaking is given.

\section{Definition and classification of mechanical symmetry breaking based on physical features}

Machinery refers to the instruments created by humans to meet their needs in terms of functionality, performance, and constraints based on physical laws. Symmetry, symmetry breaking, and asymmetry are universal features of mechanical products. Based on physical features, mechanical symmetry breaking can be classified into three levels, i.e., the mechanical general requirement symmetry breaking, working principle symmetry breaking, and cross-scale structure symmetry breaking.

\subsection{Definition and classification of mechanical gen- eral requirement space-time symmetry breaking}

Mechanical general requirement space-time symmetry breaking refers to the objective requirements which possess features of symmetry breaking in space-time. The subjects of symmetry breaking are product requirements, which consist of function requirements, performance requirements, and constraint requirements. The elements of symmetry breaking are the components of a product's function, performance, and constraint requirement. The concept system is shown in Fig. 1.

2.1.1 Mechanical general requirement spatial symmetry breaking

Function requirement spatial symmetry breaking refers to the objective functional requirements which have symmetry breaking features in the space. The functional requirements are expressed in terms of the processes for the conversion of inputs into outputs. Their spatial features include the spatial distributions of the inputs and outputs of materials, 


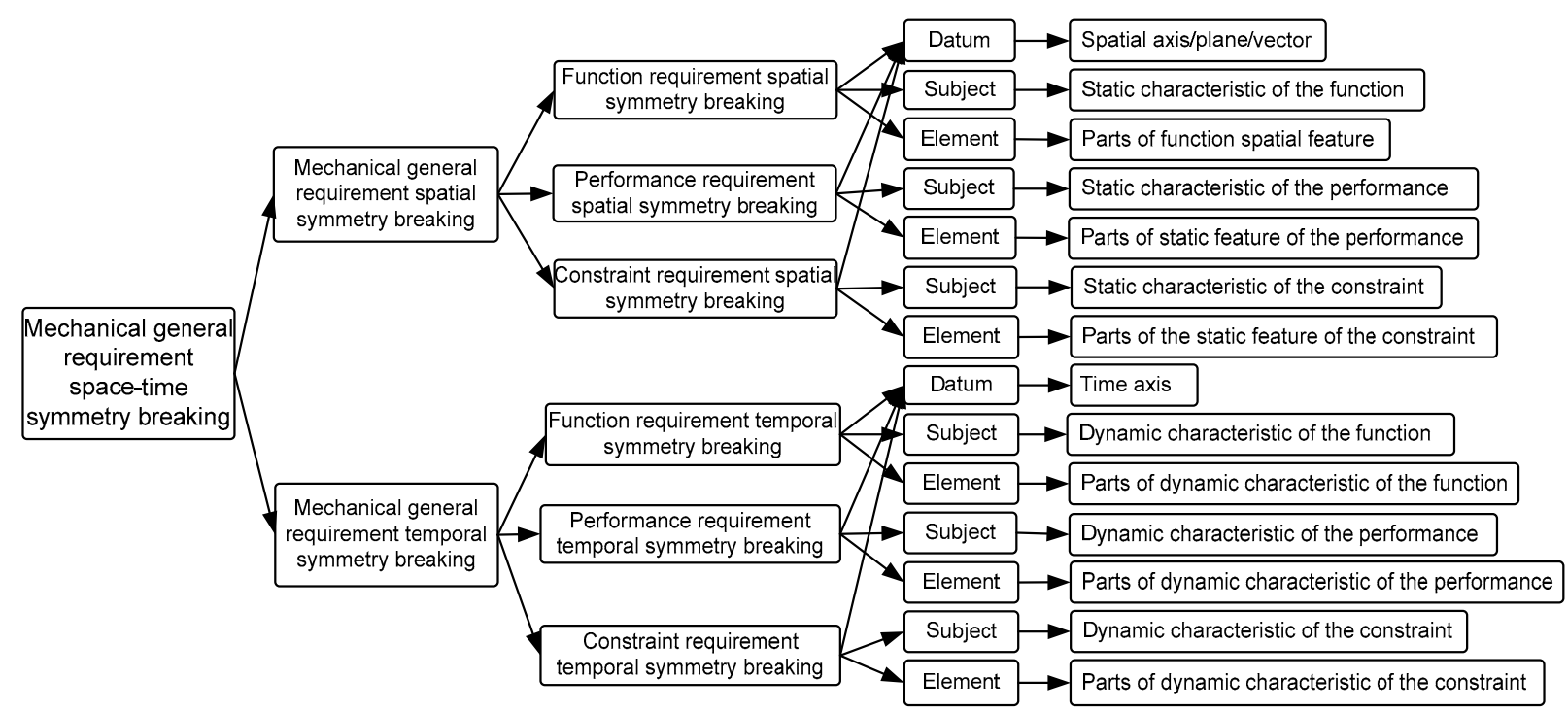

Fig. 1 Definition and classification of mechanical space-time general requirement symmetry breaking

energy or information and the working range of the functions. The umbrella shown in Fig. 2 enlarges the rear portion of the canopy while the front portion remains unchanged. Thus, the umbrella is the rotational symmetry breaking structure, the symmetry breaking datum axis is the stick, the symmetry breaking subject is the canopy, and the symmetry breaking elements are the materials of the canopy. Its effect is to realize the rotational symmetry breaking function requirements of the product, where the symmetry breaking datum is the rotational center point $O$ shown in Fig. 2 (Horgdom and Anthony, 2012), the symmetry breaking subject is the function of sheltering, and the elements are the parts of the area sheltered by the umbrella.

Performance requirement spatial symmetry breaking relates to the objective performance requirements which have symmetry breaking features in space. These performance requirements are defined as the requirements for the conversion of inputs into outputs in quantity. Their spatial features include temperature field, force field, electromagnetic field, and information field. The output light of the polarized type lens shown in Fig. $3 \mathrm{a}$ is required to follow the light intensity distribution curve shown in Fig. 3b (Liu, 2012). In Fig. 3b, if the $x$-axis is taken as the symmetry breaking datum in the space, then the symmetry breaking subject is the light intensity curve and the symmetry breaking elements are its left and right portions. To achieve this performance

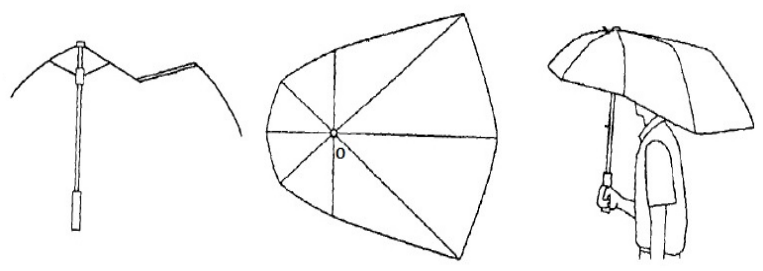

Fig. 2 Umbrella diagram (Horgdom and Anthony, 2012)

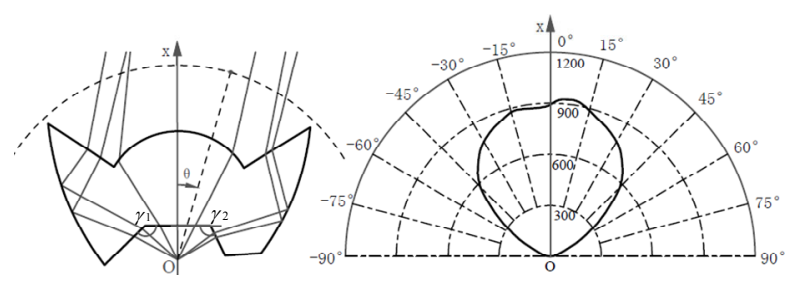

(a)

(b)

Fig. 3 Lens used in an LED (Liu, 2012): (a) lens; (b) light intensity distribution curve (unit: $\mathrm{klm}$ )

requirement, the lens is the structure mirror symmetry breaking where the symmetry breaking datum is the $x$-axis in Fig. 3a, the symmetry breaking subject is the lens, and the symmetry breaking elements are the left and right portions of the lens.

Constraint requirement spatial symmetry breaking refers to the static features of constraint requirements. For example, the restrictions on the dimensions and volume of a structure are symmetry breaking. The dimensions of the radiator depicted in Fig. 4 
(Li, 2006) should not exceed the range of the circuit board to avoid interference with other electronic components. The left part of the radiator should not exceed the area $S_{1}$ of the printed circuit board (PCB) and the right part should not exceed the area $S_{2}$ of the PCB. This constraint requirement breaks symmetry in space, where the symmetry breaking datum is the $y$-axis, the symmetry breaking subject is the area of $\mathrm{PCB}$, and the symmetry breaking elements are the areas $S_{1}$ and $S_{2}$. The structure of radiator is also mirror symmetry breaking, where the symmetry breaking datum is the $y$-axis, the symmetry breaking subject is the radiator, and the symmetry breaking elements are the left and right parts of the radiator.

2.1.2 Mechanical general requirement temporal symmetry breaking

Functional requirement temporal symmetry breaking means the dynamic characteristic of the objective function has a feature of symmetry breaking. The symmetry breaking subject is the dynamic

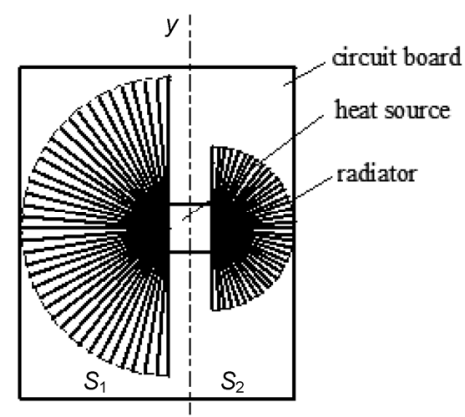

Fig. 4 Diagram of radiator $(\mathrm{Li}, 2006)$ feature of the function. Performance requirement temporal symmetry breaking means the process of an objective performance requirement changing with time has a feature of symmetry breaking. Dynamic features of performance requirements usually include time-varying curves of displacement/angular displacement, velocity/angular velocity, acceleration/ angular acceleration, and force/torque. Constraint requirement temporal symmetry breaking refers to the restriction of some dynamic processes of a system, such as displacement, velocity, force/torque, and temperature. For example, the demanded load operation time is longer than the no-load return time for most operating cycles.

\subsection{Definition and classification of working prin- ciple space-time symmetry breaking}

The solution of a working principle generally includes the working effect, working geometry, working motion, and working material that are required to achieve a function. Essentially, they are the mechanical and physical feature parameters. Working principle space-time symmetry breaking means that the compositions of a solution have symmetry breaking features in the space or time datum. The spatial features of a working principle include working geometry, motion trajectory, the range of working motion, and the spatial distribution of material. The temporal features of a working principle include the temporal attributes of the working motion or working process. The concept system is shown in Fig. 5.

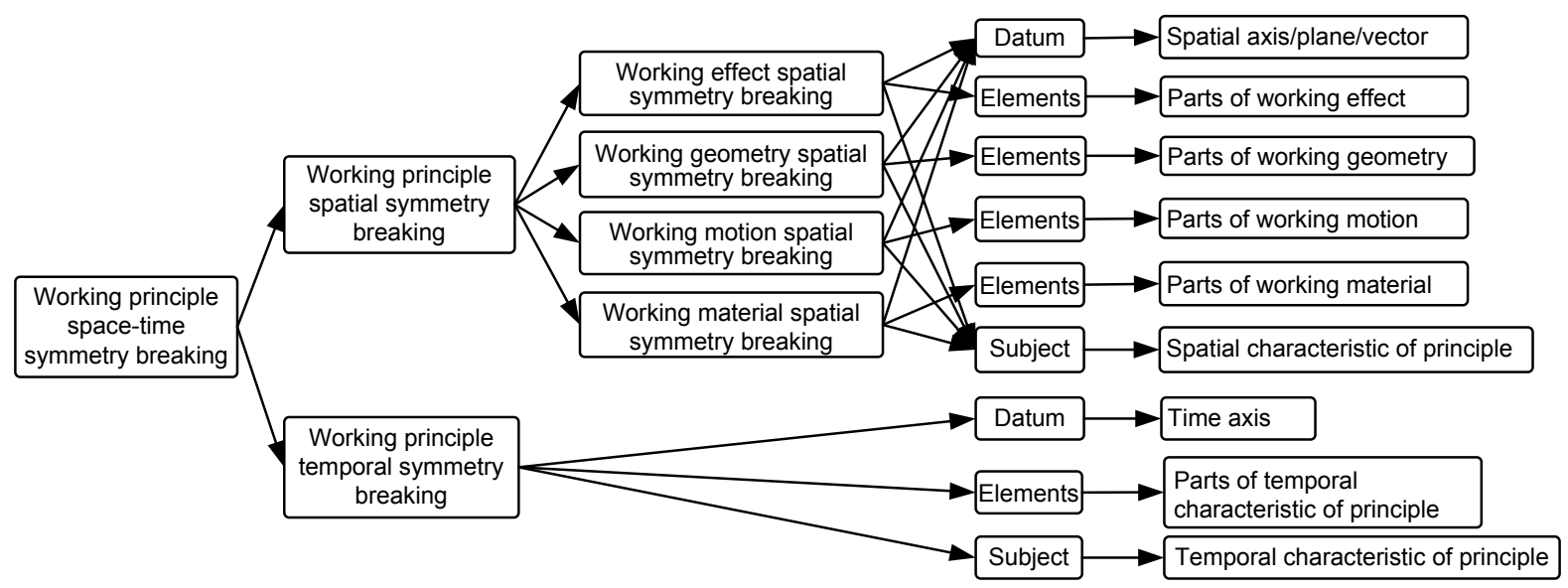

Fig. 5 Definition and classification of working principle space-time symmetry breaking 


\subsubsection{Working principle spatial symmetry breaking}

Working effect spatial symmetry breaking means one or more working effects have symmetry breaking features in the space. Common spatial features of working effects include the magnetic field distribution in electromagnetic effects or magnetic force effects, the electric current distribution in eddy current effects, and the working force distribution in force effects.

For example, since an area of the stator tooth 1 on the right side has been cut off as shown in Fig. 6, the single phase brushless direct current (DC) motor is mirror symmetry breaking in structure, where the symmetry breaking reference is the central line, the subject is the stator, and the elements are the left and right portions of the stator. The structure variation of the stator caused a variation of working principle of the motor. The magnetic field produced by the winding in the stator is mirror symmetry breaking in the space. Thus, the working effect of the motor is mirror symmetry breaking, where the datum is the central line, the subject is the electromagnetic effect, and the elements are left and right portions of magnetic field. The reason why the working principle in this case has to break its symmetry is that if the rotor was starting at the position shown in Fig. 6 (Wang et al., 2005), an imaginary symmetry designed motor would encounter a starting problem because the magnitude of the magnetic forces applied at the left and right parts of the rotor would be equal and therefore neutralized. By contrast, the starting torques of the symmetry breaking motor are not equal and there is no starting problem.

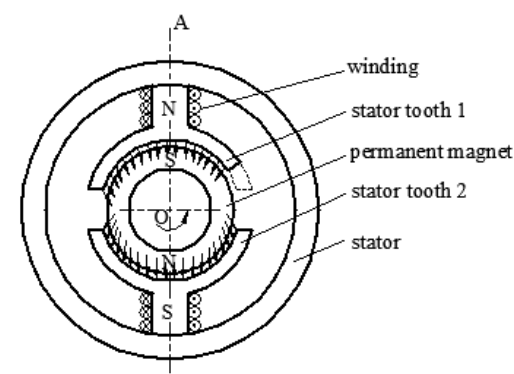

Fig. 6 Single phase brushless DC motor (Wang et al., 2005)

Working geometry spatial symmetry breaking means that the working geometry, such as points, lines, surfaces or bodies, has a symmetry breaking feature. Working geometry spatial symmetry breaking mostly exists in mechanical transmission apparatus or mechanisms. In this case, the working geometry generally represents the working point, line, plane or space of the working force, which is realized by common mechanical effects, such as gravity, hydraulic power/pressure effect, centrifugal effect, friction effect, Hooke effect, and thermal expansion effect.

As shown in Fig. 7 (Huang and $\mathrm{Xu}, 1993$ ), two fragile workpieces need to be gripped. In order to avoid the damage to them, the force applied on them must be an action plane (working geometry). For workpiece 1 , the action plane is the combination of two identical cylinder surfaces with the same parameters. In this case, the work geometry possesses mirror symmetry, of which the datum is the central line, the subject is the working plane, and the elements are two identical cylinder surfaces. For workpiece 2, the action plane is the combination of a curved surface and a plane. In this case, the work geometry is mirror symmetry breaking, of which the datum is the central line, the subject is the working plane, and the elements are the curved surface and the plane. The clamping mechanism designed for workpiece 1 has two identical springs. The structure of the clamping mechanism is also mirror symmetry. The symmetry datum is the central line, the subject is the clamping mechanism, and the elements are two fingers. The clamping mechanism for workpiece 2 is mounted on two distinct springs: a flat spring and a bent spring. Moreover, its structure is mirror symmetry breaking, where the datum is the central line, the subject is the clamping mechanism, and the elements are fingers 1 and 2.

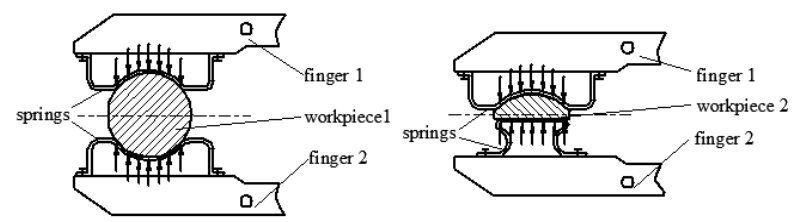

Fig. 7 Clamping of two workpieces (Huang and $\mathrm{Xu}$, 1993)

Working motion spatial symmetry breaking means the trajectories of motion or the range of working motion have a symmetry breaking feature. 
For example, the lengths of the blades 2, 3, and 4 of a stirring apparatus differ from each other as shown in Fig. 8 (Pan et al., 2013); therefore, the ranges of the working motion of each blade also vary. The working motion is translational symmetry breaking, where the symmetry breaking datum is the $y$-axis, the symmetry breaking subject is the range of the working motion, and the symmetry breaking elements are the working motions of the blades. The symmetry breaking design of this stirring apparatus causes many different eddies which increase the stirring efficiency.

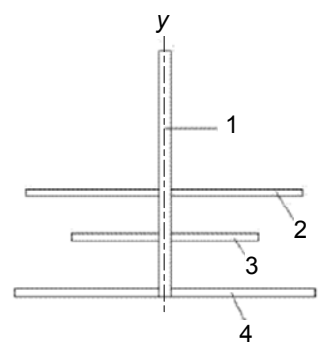

Fig. 8 Stirring shaft (Pan et al., 2013)

1: stirring shaft; $2,3,4$ : blade

Working material spatial symmetry breaking means the spatial distribution of the working material has symmetry breaking features. Working material symmetry breaking makes use of the different physical characteristics of different materials to achieve function or performance requirements. The common physical characteristics include density, elastic modulus, damping coefficient, friction coefficient, thermal conductivity, thermal expansion coefficient, and relative permeability. For instance, the structure shown in Fig. 9 (Yang, 1998) is comprised of a bolt and a sleeve. The sleeve is made by two kinds of materials having different coefficients of thermal expansion. For the sleeve with length $l_{1}$, the coefficient of linear expansion in axial direction is $\alpha_{1}$, while the coefficient is $\alpha_{2}$ for the sleeve with length $l_{2}$. Hence, the sleeve is translational symmetry breaking where the symmetry breaking datum is the axis, the subject is all the material, and the elements are the materials of the two parts. The bolt with the coefficient of $\alpha_{3}$ has the length of $l_{3}$. When the environmental temperature changes with time, only if the condition $\alpha_{3} l_{3}=\alpha_{1} l_{1}+\alpha_{2} l_{2}$ is satisfied, the distortion of the bolt is the same as the sleeve and their fitting state remains unchanged.

\subsubsection{Working principle temporal symmetry breaking}

The working principle temporal symmetry breaking means that the dynamic characteristic of a working motion or process of a principle, such as displacement, velocity, and acceleration, breaks its symmetry in the time axis. For instance, the opening and closing motion of an elevator is a linear translational motion. The velocity-time curve is shown in Fig. 10. The deceleration stage of the closing process is flatter than the opening process. It is principle temporal symmetry breaking, where the datum is the time of $t$, the subject is the velocity of the whole process, and the elements are the curves in the opening and closing stages.

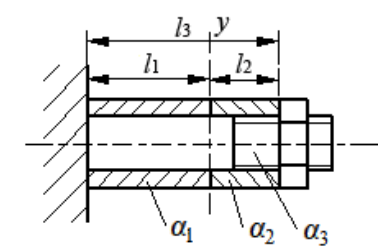

Fig. 9 Bolt and sleeve (Yang, 1998)

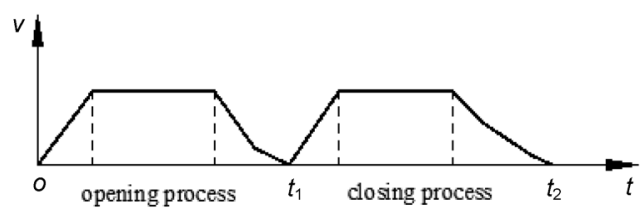

Fig. 10 Velocity curve of the elevator gates

\subsection{Definition and classification of mechanical structure symmetry breaking}

2.3.1 Definition of mechanical structure symmetry breaking

In some cases, a structure with single symmetry needs to be changed. Thus, it is no longer invariant under a symmetry operation. The process is called mechanical structure symmetry breaking and the changed structure is called a symmetry breaking structure.

Since the symmetry breaking operation is performed on the basis of the original symmetry datum, the symmetry breaking datum remains the same. The transformed elements under the symmetry operation cannot completely coincide with the original ones.

For instance, the jump ring applied to a connecting link is assembled as shown in Fig. 11a (Mi 
and $\mathrm{Pi}, 2012)$. The original design of the jump ring is shown in Fig. 11b (Mi and Pi, 2012). The structure presents mirror symmetry, where the datum line is the central line $x_{1}$, the subject is the jump ring, and the elements are the two sides of the jump ring. When used in a motorcycle that is frequently accelerated and decelerated, the jump ring under cyclic loading conditions will fail due to the reduction of the tightening force. The redesigned jump ring is shown in Fig. 11b. The depth of the groove $b$ and the dimension $a$ get smaller. Thus, the tightening force of the jump ring increases, and the service life is extended accordingly. Its structure is mirror symmetry breaking. (a)

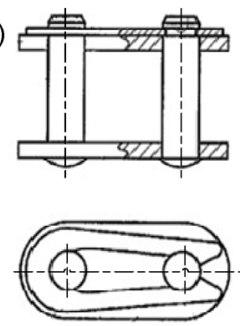

(b)

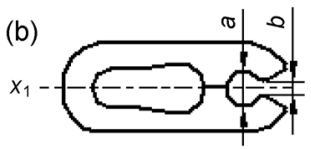

Original design

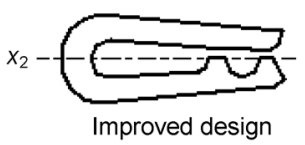

Fig. 11 Jump ring applied to a connecting link (Mi and Pi, 2012): (a) connecting link; (b) jump ring

2.3.2 Classification of mechanical structure symmetry breaking

The subject of mechanical structure symmetry breaking can concern a part or the assembled components. Thus, according to its level, structure symmetry breaking can be classified into part symmetry breaking and component symmetry breaking.

According to the different symmetry operations, symmetry breaking can be classified into rotational symmetry breaking, mirror symmetry breaking, and translational symmetry breaking.

The datum of part and component symmetry breaking include rotational central point, central line or plane, and vector. The subject of component symmetry breaking is the components themselves, the symmetry breaking elements are the composition of the components. The subject of part symmetry breaking is the part, and the symmetry breaking elements are the geometrical elements of the part. It can also be extended to the machine and series. This is not shown in the definition and classification of mechanical structure symmetry breaking in Fig. 12.

\subsubsection{Formation mechanism of structure symmetry} breaking

For the basic three types of symmetry, the symmetry structure is formed by elements repeating themselves regularly along the datum. If identical elements are repeated at equal intervals along a circle, a rotational symmetry structure is formed. If identical elements are repeated at equal intervals along a line, a translational symmetry structure is formed. If two similar elements reflect from each other along a line or plane, a mirror symmetry structure is formed.

Symmetry breaking structure is formed on the basis of the geometrical variation of the elements of a symmetry structure, which includes three patterns:

(1) Variation of the form/dimensional parameters of the element;

(2) Variation in the positional parameters of the element;

(3) Variation in the form and positional parameters of the element.

The pin hole in the lid of a gearbox is machined with the pin hole in the gearbox housing. To ensure the accuracy of assembly, the lid should not be able to be assembled reversely when mounted on the gearbox housing. Therefore, the lid is designed as a rotational symmetry breaking structure where the datum is the central point, and the symmetry breaking elements are the pin holes. As depicted in Fig. 13, the pattern of Fig. 13a shows the element positional parameter variation, the pattern of Fig. 13b shows the element form/dimension parameter variation, and the pattern of Fig. 13c shows both the element form and the position parameter variations. In practice, evaluation and selection of these patterns can be made according to the specific requirements of a design task.

\section{Correlations among mechanical general requirements, working principle, and struc- ture spatial symmetry breaking}

The concept systems of mechanical space-time symmetry breaking proposed above are classified 


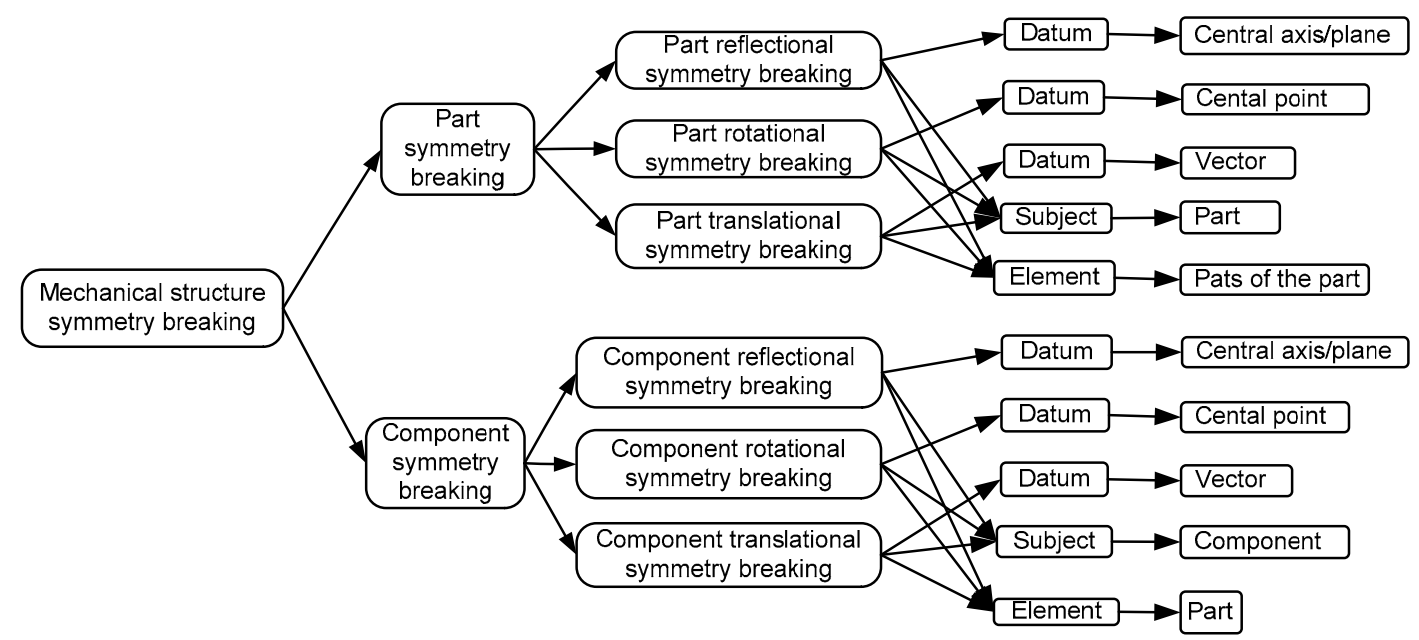

Fig. 12 Definition and classification of mechanical structure symmetry breaking

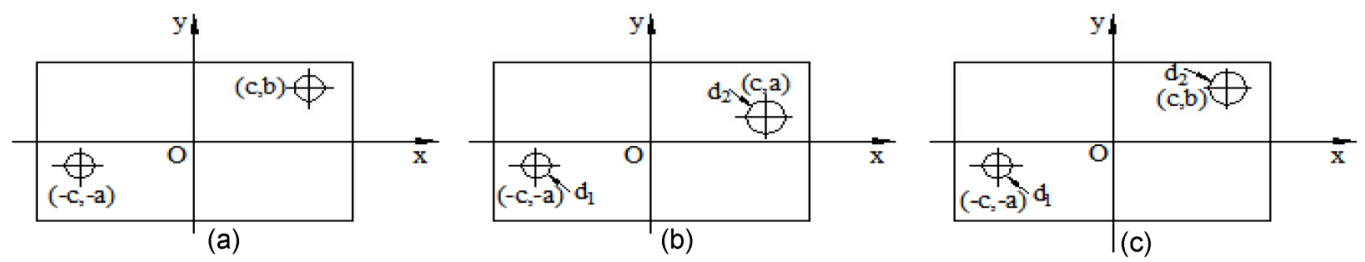

Fig. 13 Different symmetry breaking patterns of the lid of a gearbox: (a) element positional parameter variation; (b) element form/dimension parameter variation; (c) element form and position parameter variation

according to the different properties of the symmetry breaking subjects in each phase of the design process. At the beginning of product design, the general requirements for symmetry breaking are general requirements in the requirement analysis phase. The subjects of working principle symmetry breaking are working principles for the principle design phase. The subjects of structure symmetry breaking are structures in the structure design phase. Product requirements are realized by working principles, while working principles are eventually realized in product structures. The mechanical general requirement for symmetry breaking comes from the diversity of human life. Mechanical principle symmetry breaking is the means of realizing mechanical general requirement symmetry breaking while mechanical structure symmetry breaking is the physical support for the realization of mechanical working principle symmetry breaking. The symmetry breaking hierarchy through requirement and principle to structure is a process from an abstract to a concrete state and a mental to a physical one. It is also the process of the physical parameters from the macro-to-micro transformation. The partial correlation analyses are as follows.

\subsection{Correlation between the general requirement spatial symmetry breaking and the principle and structure spatial symmetry breaking}

1. Because environmental constraints constantly disturb the performance of a system, spatial symmetry breaking of a constraint requirement typically leads to the symmetry breaking of a performance requirement. As a coping strategy, if the symmetry of structure or principle breaks, then the symmetry of performance requirements might be kept. As an example, the apparatus shown in Fig. 14 is used for quenching workpieces. The depth of the hardened layer is required to be distributed uniformly over the surface of the parts, and thus its performance requirement is spatial symmetry breaking. The coils in Fig. 14a are evenly spaced. Therefore, its structure presents translational symmetry breaking, where the datum is the axis, the subject is the inductor, and the elements are the coils. The principle also presents translational symmetry breaking, where the datum is 
the axis, the elements are the eddies produced by the coils, and the subject is the eddy effect. In fact, constraint requirement symmetry breaking exists in the surrounding environment of the system. The heat radiated from the two ends is greater than that radiated from the middle part, which leads to a nonuniform distribution of temperature. Thus, the depth of the hardened layer at the two ends is shallower than that of the hardened layer in the middle part. Accordingly, the performance requirement is symmetry breaking in the space. The redesigned inductor is shown in Fig. 14b. The spacing of the coils at the two ends has been decreased, so that the inductor can generate more heat there thus giving a uniform temperature distribution. As a result, the symmetry of performance requirement is obtained. The redesigned structure presents translational symmetry breaking, where the datum is the axis, the subject is the inductor, and the elements are the coils. The principle also presents translational symmetry breaking, where the datum is the axis, the elements are the eddies produced by the coil, and the subject is the eddy effect.

2. When a constraint requirement is symmetrical, or there is no constraint requirement, principle spatial symmetry may generally be used to obtain performance requirement spatial symmetry, and principle spatial symmetry breaking may be used to obtain performance spatial symmetry breaking. As shown in Fig. 15, in order to quench a gear with large tooth width, double coils were used. The surrounding heat dissipation environment of the coils is mirror-symmetrical (constraint is also symmetrical). Thus, the principle and structure of the apparatus are mirror-symmetrical.

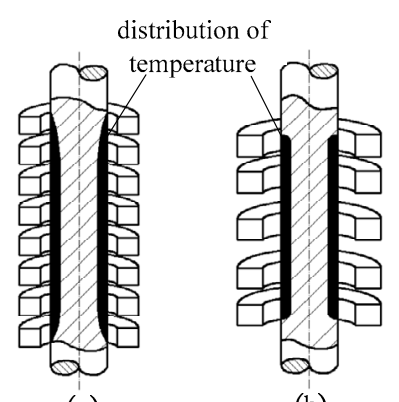

(a)

Fig. 14 Induction hardening system for spindles: (a) coils evenly distributed; (b) redesigned inductor (decrease the spacing of the coils at the two ends)

Two workpieces in Fig. 16 (Ding, 2012) are supposed to be quenched with a uniform hardened layer over their surfaces. For the workpiece 2 in Fig. 16a, the distribution of the hardened layer on the left is identical with the one on the right. Thus, the performance requirement of the inducing conductor 1 is mirror-symmetrical, where the datum is the $y$ axis of the fixing piece 3 , the subject is the hardened layer, and the elements are the hardened layers. The workpiece 2 in Fig. 16b has a groove on its left side. The distribution of the hardened layer on the left is not completely identical with the one on the right. Thus, the performance requirement is mirror symmetry breaking, where the datum is the $y$-axis, the subject is the hardened layer, and the elements are the left and right parts of the hardened layer.

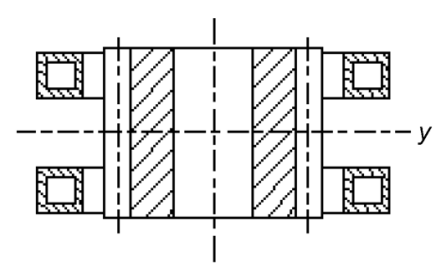

Fig. 15 Induction hardening system for gears

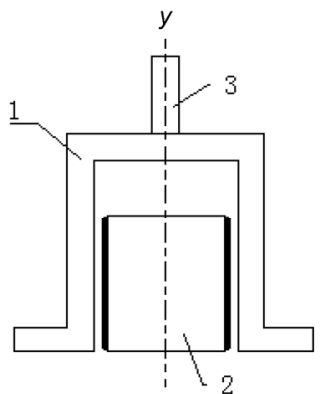

(a)

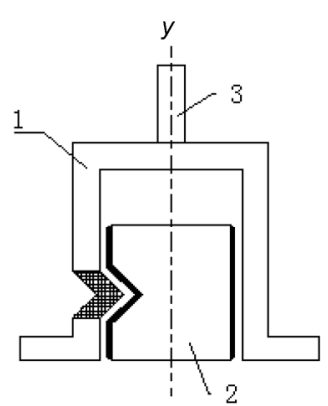

(b)
Fig. 16 Induction hardening system (Ding, 2012)

\subsection{Correlation between general requirement symmetry breaking and structure spatial sym- metry breaking}

Generally, structure spatial symmetry breaking is used to achieve principle spatial symmetry breaking, and principle spatial symmetry breaking is used to achieve function/performance spatial symmetry breaking. Therefore, structure spatial symmetry breaking is a final way of achieving function/ performance spatial symmetry breaking. 
Constraint requirement spatial symmetry breaking can be achieved by structure symmetry breaking. As shown in Fig. 17 (Zhou et al., 2012), the width of a hoistway $S$ restricts the opening range of the double-gate elevator. Namely, the total width of the left and right gates $a+b$ cannot exceed $S_{1}+S_{2}$, and $S=a+b+S_{1}+S_{2}$. Due to the spatial symmetry breaking restriction on the position of the gate, $S_{1}>S_{2}$ must be satisfied. To ensure the gates can be opened without any interference, the condition $a>b$ must be satisfied. Besides, the width of the left gate approaches but not exceeds $S_{1}$, and the width of the right gate approaches but not exceeds $S_{2}$. Consequently, the gates of the elevator are spatial mirror symmetry breaking, where the datum is the $y$-axis, the subject is the double gate, and the elements are the left and right gates.

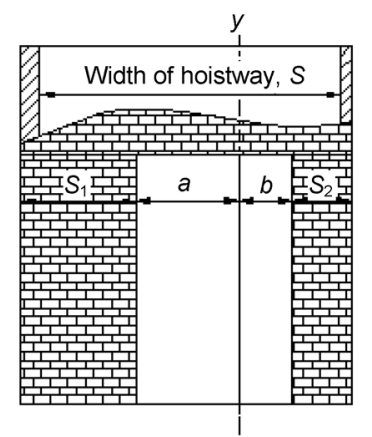

Fig. 17 Configuration of a double-gate elevator (Zhou et al., 2012)

\section{Regular knowledge on the application of symmetry breaking in design}

The basic characteristic of a symmetry breaking structure is that the geometrical parameters of different elements are distinct from each other. Structure symmetry breaking generally utilizes the geometrical differences of the symmetry breaking elements to satisfy the function, performance, and constraint requirements of a product. On the basis of the analysis of hundreds of design cases, regular knowledge about utilizing various symmetry breaking patterns and their effects to satisfy typical requirements of products are concluded and extracted. These results will raise the awareness and efficiency of applying symmetry breaking knowledge to solve design problems.
4.1 Using symmetry breaking to satisfy functional requirements of products

Principle 1: the principle for achieving the variable speed function

The speed of a transmission apparatus will be varied if it has structure symmetry breaking. For example, the structures of non-circular gears, sprockets, and pulleys are all rotational symmetry breaking, where the datum is the rotational central point, the subject is the entire structure, and the elements are the teeth. Because the positional parameters of some elements are different from others, the related instant transmission ratios are different, and the instant output angular velocities are different, too. Thus, transmission apparatus with structure symmetry breaking has achieved a variable speed function.

\subsection{Using symmetry breaking to satisfy perfor- mance requirements of products}

Principle 2: the principle for reducing the output fluctuation of a transmission system

If the dynamic output characteristic of each symmetry element is in phase, the output fluctuation of a system will be reduced by making a phase difference for each element, i.e., changing the dimension, form, or position parameters properly.

As shown in Fig. 18a (Breton et al., 2000), the permanent magnets of the motor are arranged with rotational symmetry. The cogging torque resulted from the interaction of permanent magnet fields and electric current fluctuates periodically. The electromagnetic torque resulted from the magnet field and electric current inter-action is a constant value. The total output torque of the motor fluctuates. By changing the arrangement of magnets, as shown in Fig. 18b (Breton et al., 2000), the magnets 2, 3, and 4 are rotated somewhat, and, as a result, structure symmetry breaks, where the datum is the central point, and the subject is the magnet. Then, principle symmetry breaks, where the subject is the magnetic field, and the element is each magnetic field. The deflection angle of each magnet is different, so the phase of the cogging torque is different as well. As long as the parameters are properly chosen, the fluctuation of the composite cogging torque can be reduced. The contrast of two cogging torque curves is shown in Fig. 18c. 


\section{Principle 3: the principle for reducing collision and impact}

The collision and impact between parts that are subjected to uniform loads can be reduced by breaking their symmetry. Because the forces exerted on the elements are different, unbalanced forces or torques will be produced.

As illustrated in Fig. 19 (Lan and Liu, 2010), the piston of the engine collides with the wall of the cylinder due to the existing gap. The pin of the piston is on the central plane of the piston, and the structure of the piston is mirror-symmetrical. When the piston moves upward, the piston leans against the right side of the wall. When the piston moves across the top dead center, the direction of the force changes and the piston moves from one side to the other side. As a result, a collision occurs. The piston boss shown in Fig. 19b is deviated with a distance of 1$2 \mathrm{~mm}$ from the left, and thus the pressure applied on the right part of the piston is larger than that on the left. Consequently, the piston is subjected to a torque.
When the piston is about to change its direction of movement, it rotates clockwise around the pin. In this case, the piston skirt contacts the other side of wall first, and then the piston head makes contact. The two-step contact process will reduce the impact of collisions and thus the noise and vibration of the motor. At the structure level, the structure of the piston is mirror symmetry breaking, where the subject is the piston, and the datum is the central line. At the principle level, the principle is working geometry symmetry breaking. The datum is the point $O$. The dimensions of the working lines are different.

Principle 4: the principle for reducing system noise I

Changing positional parameters of elements appropriately will make a phase difference in noise waves. As a result, noise waves with different phases will interfere and cancel each other, and the total noise will be reduced.

Tire tread pattern generates a strong noise in contact with the road. Fig. 20 (Huang, 2008) shows

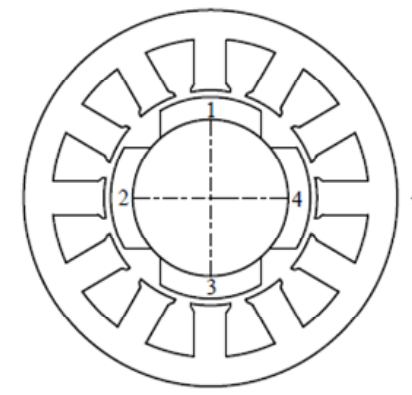

(a)

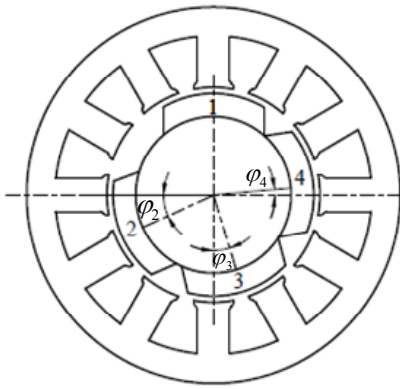

(b)

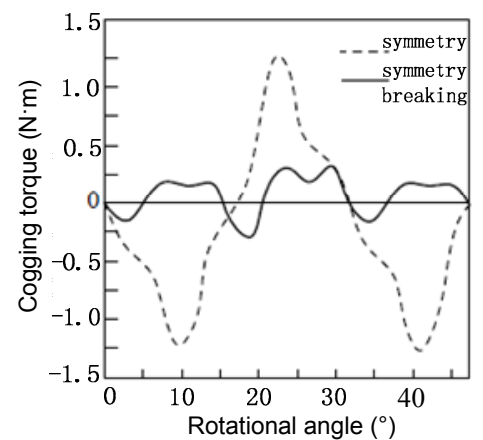

(c)

Fig. 18 Permanent magnetic motor (Breton et al., 2000)

(a) Rotational symmetry; (b) Rotational symmetry breaking; (c) Cogging torque curves

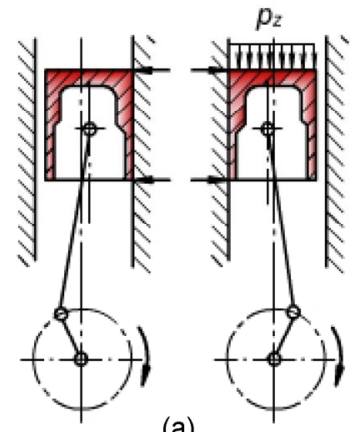

(a)

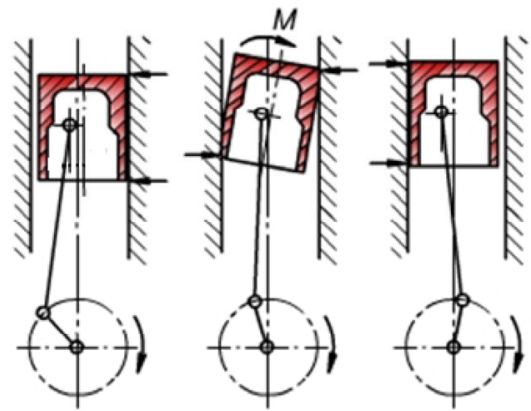

(b)

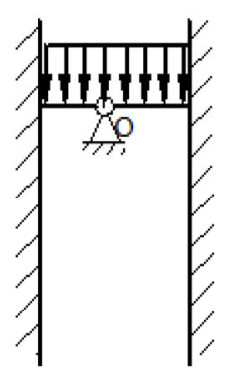

(c)

Fig. 19 Motion processes of two types of engine (Lan and Liu, 2010)

(a) Normal engine; (b) Deviated pin engine; (c) Principle symmetry breaking 
two types of tread patterns. The tread in Fig. 20a is mirror-symmetrical about the central line $A_{1} A_{2}$, and the elements are the upper and lower tread patterns. As seen from the time-domain waveform diagram, the sound frequencies, amplitudes, and phases of elements are the same. The sound pressure level (SPL) of the superimposed sound wave becomes larger, and the tire noise becomes stronger. The tread pattern in Fig. $20 \mathrm{~b}$ is arranged in a staggered pattern, which is mirror symmetry breaking. The phases of the noise waves generated by the upper and lower patterns are different and partially counteract. The peak value of the superimposed sound wave is much smaller than that from the tire pattern in Fig. 20a. As a result, noise is reduced. In this case, no extra apparatus is used to reduce the noise. Compared with commonly used noise elimination techniques, symmetry breaking design of the thread pattern is very economical.

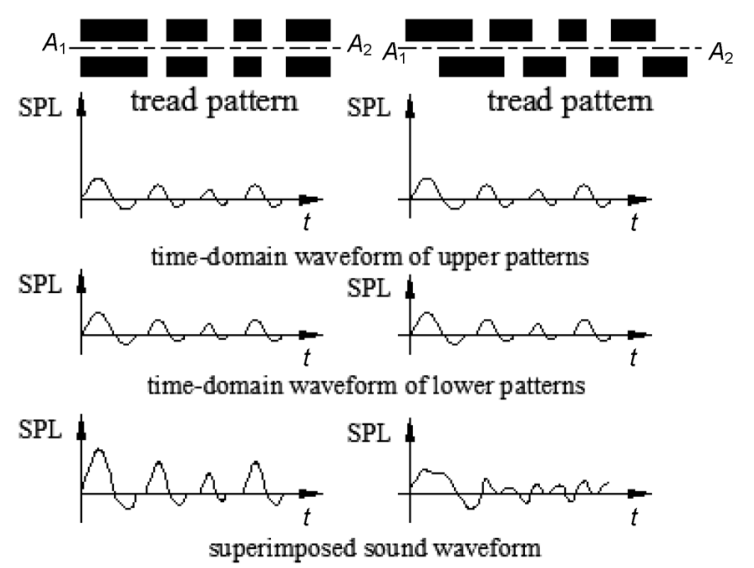

(a)

(b)

Fig. 20 Tire noise waves (Huang, 2008): (a) symmetrical tread pattern; (b) staggered tread pattern

\section{Principle 5: the principle for reducing system noise II}

The sound frequency and magnitude generated by symmetrical elements are approximate, while the sound frequency and magnitude generated by symmetry breaking elements are quite different. Thus, a symmetry breaking design can reduce noise level.

As shown in Fig. 21b (Todd, 2011), for a chain drive system with rotational symmetrical sprockets, the engagement process of sprocket tooth and rollers is almost the same, so the impact noise is strong. By modifying the positional parameters of the sprocket grooves, as shown in Figs. 21a and 21c (Todd, 2011), the sprocket has three different root radii $R_{1}, R_{2}$, and $R_{3}$ arranged in a rotational symmetry breaking pattern. When rollers engage with the sprocket, the impact distance and impact force are different. The sound frequencies are modulated and the noise is decreased.

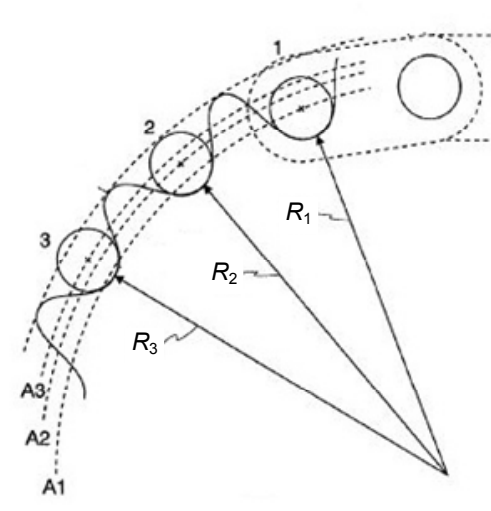

(a) A sprocket having three different root radii

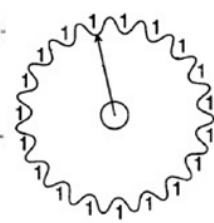

(b) Rotational symmetry sprocket

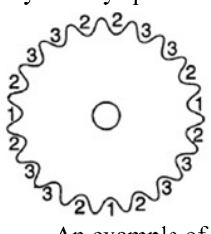

An example of

(c) distribution of different root radii in sprocket
Fig. 21 The sprocket with different root radii (Todd, 2011)

\section{Principle 6: the principle of load match}

When a structure-symmetrical system is subjected to asymmetrical loads, the element with the larger load can be broken and its bearing capacity made to match the load.

As shown in Fig. 22a (Guo et al., 2011), the bearings used in the spindle of a wind turbine are tapered roller bearings which are mirror-symmetrical about the $y$-axis. They are configured in the fixed end of the spindle and are capable of withstanding bi-directional axial loads. Usually, the load in a wind turbine is large, and most of the force goes towards the right. Therefore, most of the load is allocated to the bearing $\mathrm{A}$. The radial loads of bearings $\mathrm{A}$ and $\mathrm{B}$ are the same. Thus, bearing A will fail first and then the entire bearing will fail. In the bearing shown in Fig. 22b (Guo et al., 2011), the contact angle of bearing $\mathrm{A}$ is larger than that of bearing $\mathrm{B}$, so bearing A has a relatively high axial load carrying capacity while bearing $\mathrm{B}$ has a relatively high radial load carrying capacity. Meanwhile, the sphere centre $O_{1}$ of the ring is deviated from centre $\mathrm{O}_{2}$ of the bearing, and thus more radial load is allocated to bearing $\mathrm{B}$ and more axial load is allocated to bearing A. 
Compared with the structure in Fig. 22a, the symmetry breaking structure in Fig. 22b makes full use of the load carrying capacities of bearings A and B, and the life-circle of both bearings is increased.

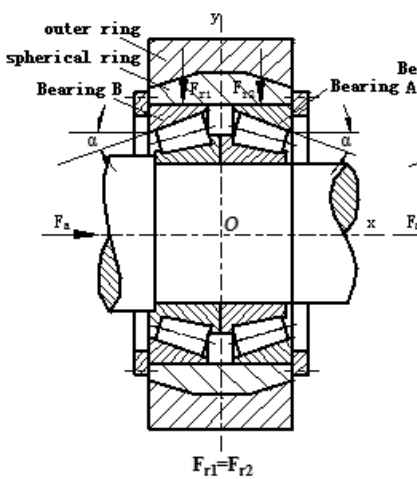

(a)

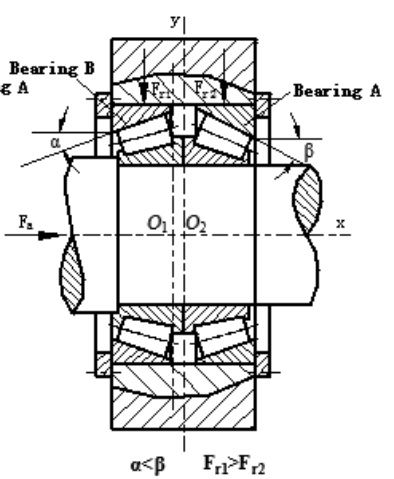

(b)
Fig. 22 Tapered roller bearings (Guo et al., 2011)

(a) Symmetrical type; (b) Symmetry breaking type

\subsection{Using symmetry to satisfy the constraint re- quirements of products}

\subsubsection{To satisfy the constraint of assembly}

\section{Principle 7: the principle of increasing identifica- tion capability}

Utilizing the geometrical difference of symmetry breaking elements will make the non-geometrical feature of a part easy to identify.

For a symmetry part, assume there are some features that are hard to distinguish by appearance, but have to be distinguished at the assembly stage. One way of realizing the requirements of easy identification during assembly is to replace the related symmetrical elements with symmetry breaking ones to do the same job. By doing this, the nongeometrical difference of related elements is converted into a geometrical difference. For example, in the pin shown in Fig. 23 (Pahl and Beitz, 1992), its upper part is hardened, while the lower part is not. By breaking the mirror-symmetrical structure of the original design (Fig. 23a), the identification and thus the accurate location of the newly designed version is much easier (Fig. 23b).

\section{Principle 8: the principle of positioning during assembly}

Symmetrical parts will be fitted in their unique assembly position if structure symmetry breaking is used to avoid wrong assembly.
As shown in Fig. 24 (Chen and $\mathrm{Wu}, 2003$ ), the bearing cap of the crankshaft and the bolts of the cylinder body are connected together to bore the hole of crankshaft first. In the following step, the bearing cap has to be removed to mount the crankshaft. During this process, there is a high possibility that the bearing cap will be mounted oppositely, causing damage to the precision of the hole and the operation of the crankshaft. To avoid this, the distance from one side of the bearing cap to the center line $\mathrm{O}_{1} \mathrm{O}_{2}$ differs from the other side, while the distances from the center line of each bolt to the center line $O_{1} O_{2}$ are identical. Consequently, the structure is mirror symmetry breaking about the center line $O_{1} O_{2}$. In order to be mated with the bearing cap, the structure dimensions in the cylinder body are modified accordingly. If the bearing cap were mounted oppositely, as shown in Fig. 24b, the bolts would interfere with the cylinder body and wrong assembly would be prevented.

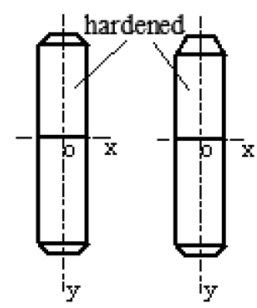

(a)

(b)

Fig. 23 Examples of increasing identifiability (Pahl and Beitz, 1992): (a) original design; (b) improved design

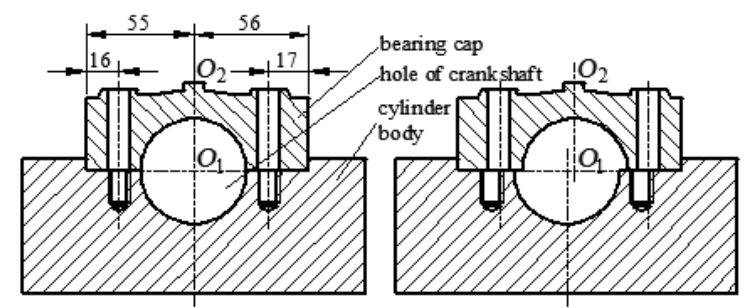

(a)

(b)

Fig. 24 Bearing cap of a crankshaft (Chen and Wu, 2003): (a) correct assembly (unit: $\mathrm{mm}$ ); (b) wrong assembly

\subsubsection{To satisfy the constraint of non-interference}

Due to location differences among the elements, if there is a possibility that one of the elements interferes with surrounding objects, then the structure dimensions of the element should be restricted. 
Because other elements are not restricted, structure symmetry breaking occurs. For example, a clamping apparatus for a declination rectification and guidance device is shown in Fig. 25 (Liu, 2013). This device is mounted on a boat. Because one clamp arm is located near the side of the boat, the arm can easily collide with the boat when it rotates. To avoid this, this arm is shortened. Therefore, the structure of the clamping device is mirror symmetry breaking.

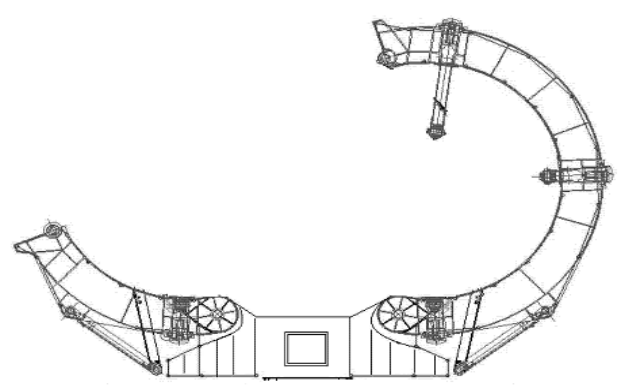

Fig. 25 Clamping apparatus of a declination rectification and guidance device (Liu, 2013)

4.3.3 To satisfy the constraint of dimensions or volumes

Assuming that a product meets requirements, the smaller its structure is, the better it will be. On one hand, it will save materials, reduce manufacturing costs, and increase the economy of the product. On the other hand, especially for moving parts, it will decrease inertia and improve dynamic performance. Structure symmetry breaking can satisfy the constraint of volume reduction generally, because the margins for reducing dimensions of elements are different. Fig. 26a illustrates a settling centrifuge (Hong and Liu, 2006). Due to the centrifugal effect in the separation section, the liquid with relatively high density settles near the inner wall of the drum. Thus, the heavy liquid flows out of the heavy-liquid outlet and light liquid flows out of the axial lightliquid outlet. The drum in Fig. 26a is mirror symmetry structure. The axial dimension and the whole volume are relatively large. To diminish its volume, the length of the light liquid channel can be reduced. As shown in Fig. 26b, the dimensions of the left part of the drum are reduced in the redesigned centrifuge, while the right part remains the same.

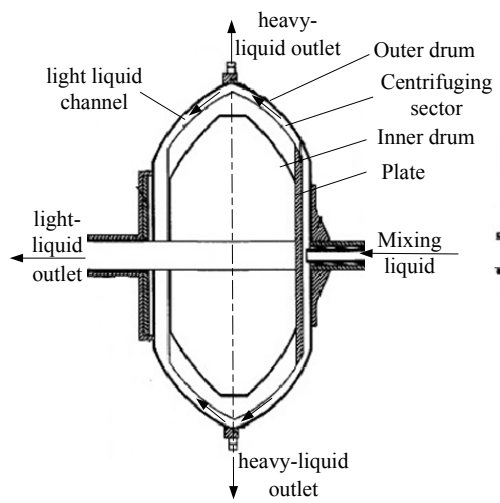

(a)

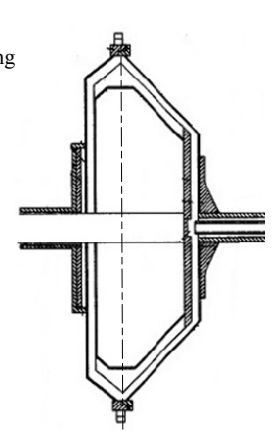

(b)
Fig. 26 A settling centrifuge (Hong and Liu, 2006) (a) Original design; (b) Redesign

\section{Application method, process, and in- stance of symmetry breaking in mechanical concept design}

\subsection{Application method and process of symmetry breaking in mechanical concept design}

Starting from design requirements, the method and process of applying design principles, guidelines, and instance knowledge for concept design are shown in Fig. 27.

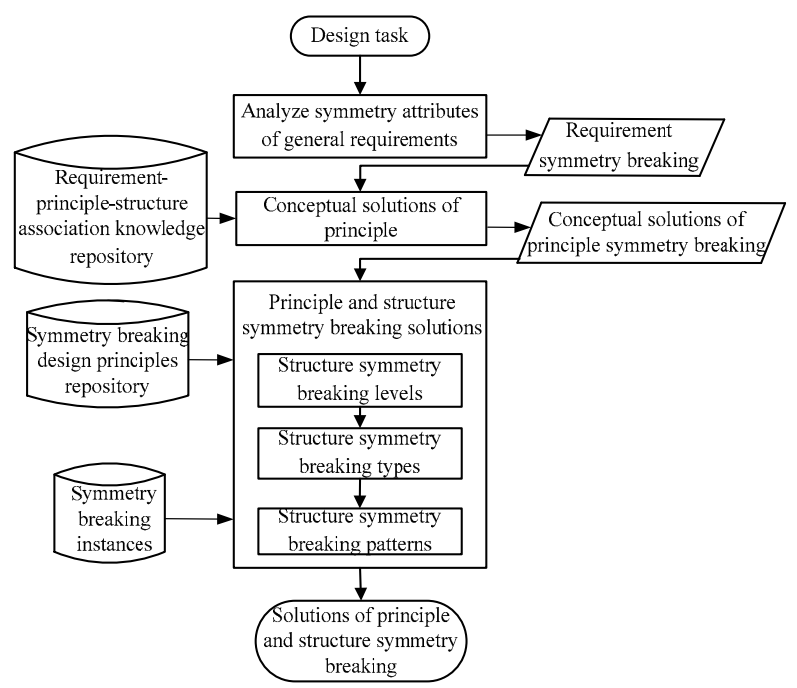

Fig. 27 Application method and process of symmetry breaking in mechanical concept design

Firstly, the general requirements of a product are specified by analyzing its design task, especially 
noting the symmetrical, asymmetrical, and symmetry breaking requirements of its functions, performances, and constraints. Then, the symmetry breaking principle and/or structure solutions can be obtained by the aid of the "requirements-principle-structure association knowledge repository" and "design principle guideline repository". Finally, variants of principle solutions in various symmetry breaking types, patterns, and structures can be found by applying the systematic variation strategy for further comparison and selection.

\subsection{Instance of symmetry breaking in a mechani- cal concept design}

The above design method and design process of symmetry breaking are illustrated with a novel chain drive system designed by the authors as follows.

Design task: a chain drive system is often used to transmit dynamically varying torque. For instance, an electric motor drives a crank-slide mechanism with a chain drive unit. Due to the inertial force generated by the non-uniform motion of the crank, the drive torque of the crank also varies dynamically. The fluctuating torque is transmitted to the chain drive unit, making the tension of the chain's tight side fluctuate. Thus, the smoothness of the chain drive is reduced. The fluctuating loads on the chain drive unit should be reduced to increase its smoothness of operation.

Analysis of structure symmetry and symmetry breaking of chain drive: the chain drive unit is a multi-hierarchy symmetry and symmetry breaking system. The symmetry features of the chain drive unit related with the objective function contain translational symmetry and rotational symmetry. The subject of the translational symmetry is the chain, and the elements are the links. The subject of the rotational symmetry is the sprocket, and the elements are the teeth of the sprocket.

Concept design of structure symmetry breaking: the sprocket and chain are selected as the symmetry subjects. The structure symmetry breaking type of the sprocket is rotational symmetry breaking, where the symmetry breaking elements are the teeth. The structure symmetry breaking type of the chain is translational symmetry breaking where the symmetry breaking elements are the chain links. As shown in Table 1, five structure symmetry breaking solutions are generated after applying principle 2 from Section 3.2 and systematically varying the form/dimension parameters and positional parameters. The solutions are listed as follows.

1. Non-circular sprocket with different pitch radii: the driven sprocket is a common non-circular sprocket. As shown in Fig. 28a, assuming that the tensile force $F$ of the chain is a constant value, the loading torque $M(\theta)$ varying with the sprocket radius is determined, i.e., $R(\theta)=M(\theta) / F$. When the fluctuation of loading torque is large, the non-circular degree is large as well, which might cause vertical vibration of the chain. Thus, the non-circular degree is limited and the processing of a non-circular contour is relatively difficult.

Table 1 Structure symmetry breaking solutions of chain drive unit

\begin{tabular}{|c|c|c|c|c|c|}
\hline Subject & $\begin{array}{c}\text { Symmetry } \\
\text { breaking type }\end{array}$ & Elements & Geometric parameter & $\begin{array}{c}\text { Structure } \\
\text { parameters of } \\
\text { elements }\end{array}$ & $\begin{array}{l}\text { Structure symmetry } \\
\text { breaking solution }\end{array}$ \\
\hline \multirow[t]{3}{*}{ Sprocket } & $\begin{array}{l}\text { Rotational } \\
\text { symmetry }\end{array}$ & Teeth & Shape parameter & Contour line & $\begin{array}{l}\text { Sprocket with differ- } \\
\text { ent contour line }\end{array}$ \\
\hline & breaking & & Location radius & Pitch radius & $\begin{array}{c}\text { Non-circular sprocket } \\
\text { with different pitch } \\
\text { radius }\end{array}$ \\
\hline & & & Location angle & Pitch & $\begin{array}{l}\text { Sprocket with } \\
\text { different pitch }\end{array}$ \\
\hline \multirow[t]{2}{*}{ Chain } & $\begin{array}{c}\text { Translational } \\
\text { symmetry } \\
\text { breaking }\end{array}$ & Chain links & Shape parameter & $\begin{array}{l}\text { Diameter and } \\
\text { length of the } \\
\text { roller }\end{array}$ & $\begin{array}{l}\text { Chain having rollers } \\
\text { of different diameters }\end{array}$ \\
\hline & & & Location parameter & Pitch & Unequal pitch chain \\
\hline
\end{tabular}




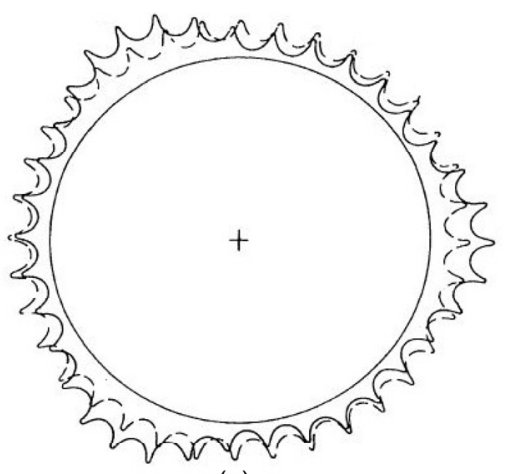

(a)

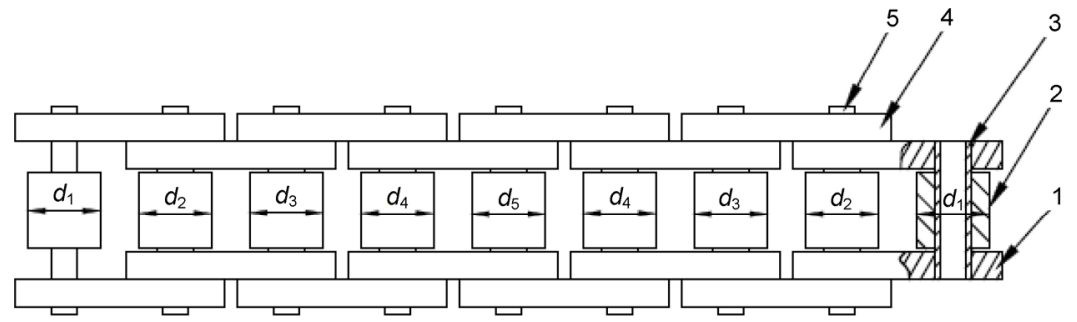

(c)

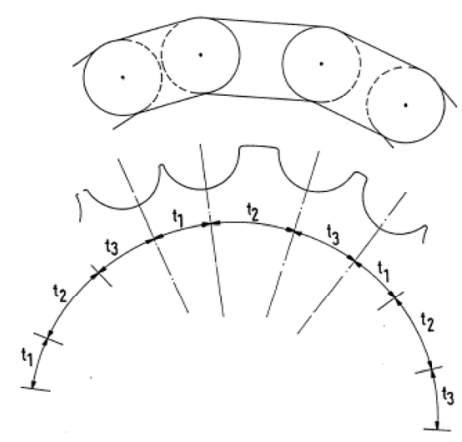

(b)
Fig. 28 Structure symmetry breaking solutions for chain drive apparatus

(a) Sprocket with unequal pitch; (b) Chain with unequal pitch and sprocket; (c) Chain having unequal diameter rollers. 1: inner link; 2: roller; 3: sleeve; 4: outer link; 5: pin

2. Unequal pitch chain drive unit: as is shown in Fig. 28b, the unit is comprised of a chain and two sprockets which have several different pitches. However, when the ratio of the pitch to the pitch circle radius is relatively large, the solution varies the actual pitch radius significantly and might cause high impact and large fluctuation.

3. Chain having rollers with different diameters: as shown in Fig. 28c, the chain is comprised of rollers with different diameters. The distance from the roller center to the center of the sprocket varies when the chain engages with the sprocket. Accordingly, the working radius of the tension force $F$ is different. On the premise that the roller radius is smaller than the curvature radius, the fluctuation of the tensile force $F$ can be reduced. For example, the number of the rollers is $N$ in one cycle. When the loading torque of roller $i(i=1,2, \ldots, N)$ engaging with sprocket is $M_{i}$, the meshing radius $R_{i}=M_{i} / F$ can be calculated. Assume that the radius of the pitch circle of the sprocket is $R$, the roller diameter $d_{i}$ can be calculated as follows: $d_{i}=2\left(R_{i}-R\right)$. The rollers can be easily processed, while the assembly is straightforward. The authors have obtained the patent of the method (Wang et al., 2013).

\section{Conclusions}

The multi-hierarchy concept systems of mechanical symmetry breaking are established and the correlations between different levels of symmetry breaking are analyzed based on the physical features from the product's general requirements (function, performance, and constraint), its working principle to its structure, and from macro to micro. Eight design principles for applying various symmetry breaking patterns and their effects in improving the product's function and performance are proposed. The method and process of applying symmetry breaking in mechanical concept design are set out. These principles, method, and process are then used in the concept design of a gear chain. The structure of the innovative gear chain has a symmetry breaking character, and, compared with the traditional ones, its transmission stability is much improved.

Based on the physical features of systems, machines, assemblies, and parts, the concept system of cross-scale symmetry breaking is also advanced. Its in-depth study needs the quantitative model of mechanical symmetry breaking. The related research results will be published in other papers. 
In addition, the concept system and effect of mechanical symmetry breaking, the application laws of symmetry breaking for mechanical products, and the integration strategies of symmetry breaking and symmetry for mechanical design are worth studying.

\section{References}

Angelo, L.G., Stefano, P.D., 2013. Bilateral symmetry estimation of human face. International Journal on Interactive Design and Manufacturing, 7(4):217-225. http://dx.doi.org/10.1007/s12008-012-0174-8

Boothroyd, G., 2009. Assembly Automation and Product Design. Translated by Xiong, Y.J., Shan, C.W., Lou, W.Z., China Machine Press, Beijing, China (in Chinese).

Breton, C., Bartolome, J., Benito, J.A., et al., 2000. Influence of machine symmetry on reduction of cogging torque in permanent-magnet brushless motors. IEEE Transactions on Magnetics, 36(5):3819-3823. http://dx.doi.org/10.1109/20.908386

Chen, S.Z., Wu, Y.F., 2003. Error-proofing's philosophies and application. The First Asian Network for Quality Congress and the 17th Asia Quality Symposium, Beijing, China, p.669-675.

Ding, X., 2012. Surface Induction Hardening Apparatus Used for V-type Crossing Pin Roller Rail. China Patent CN201210048392.X (in Chinese).

Dong, Z.G., Ni, P.G., Zhu, J., et al., 2012. Transparency window for the absorptive dipole resonance in a symmetry-reduced grating structure. Optics Express, 20(7):7206-7211. http://dx.doi.org/10.1364/OE.20.007206

Friedman, M., 2008. The evolutionary origin of flatfish asymmetry. Nature, 454(7201):209-212. http://dx.doi.org/10.1038/nature07108

Ge, X.K., Zhao, Q.X., Shao, H., et al., 2011. Research on slenderness structure vibration induced by detouring flow symmetry breaking-taking the accident of Tacoma narrow bridge damage caused by wind vibration as an example. Journal of Disaster Prevention and Mitigation Engineering, 31(4):430-435. http://dx.doi.org/10.13409/j.cnki.jdpme.2011.04.010

Guo, X.Q., Guo, Y., Wu, G.H., 2011. A Main Bearing Apparatus of the Spindle of Wind Turbine. China Patent CN201120053260.7 (in Chinese).

Hong, G.C., Liu, Y.A., 2006. A Horizontal Type Centrifugal Machine. China Patent CN200520110085.5 (in Chinese)

Horgdom, G., Anthony, H.R., 2012. An Asymmetry Umbrella Having an Improving Operating Mechanism. China Patent CN201120485752.3 (in Chinese).

Hou, S.Q., Xu, J.Q., 2015. Relationship among S-N curves corresponding to different mean stresses or stress ratios. Journal of Zhejiang University-SCIENCE A (Applied Physics \& Engineering), 16(11):885-893. http://dx.doi.org/10.1631/jzus.A1400321
Huang, G.J., 2008. Simulation Analysis of Asymmetry Tread Patterns and Study of Its Optimization Software. MS Thesis, Wuhan University of Technology, Wuhan, China (in Chinese).

Huang, Y.P., Xu, J.J., 1993. Automation Mechanism Design Illustrated Sourcebook. China Railway Press, Beijing, China (in Chinese).

Lan, Y.X., Liu, Z.F., 2010. The influence of a biased piston pin on pin's second motion. Internal Combustion Engine and Part, 1:11-13.

http://dx.doi.org/10.3969/j.issn.1674-957X.2010.01.005

Li, X.Z., 2006. A Radiator. China Patent CN200510103968.8 (in Chinese).

Liu, L.W., 2012. A Polarized Lens Used in LED Light. China Patent CN201110324379.8 (in Chinese).

Liu, T.W., 2013. Pile Body Centralizer and Rectification System. China Patent CN201310163710.1 (in Chinese).

Masoumi, A., Pellicano, F., Samani, F.S., et al., 2015. Symmetry breaking and chaos-induced imbalance in planetary gears. Nonlinear Dynamics, 80(1-2):561-582. http://dx.doi.org/10.1007/s11071-014-1890-3

Mi, G.Z., Pi, Z.J., 2012. A Jump Ring Used in Chain and a Chain Using the Jump Ring. China Patent CN200980159276.1 (in Chinese).

Pahl, G., Beitz, W., 1992. Engineering Design. Translated by Zhang, Z.M., China Machine Press, Beijing, China (in Chinese).

Pan, X.J., Song, J.J., Chen, Q.Y., et al., 2013. A Multi-level Stirrer. China Patent CN201310327951 (in Chinese).

Rajasri, I., Gupta, A., Rao, Y.V.D., 2014. Symmetry and its effects on structures of planetary gear trains. Journal of the Institution of Engineers (India): Series C, 95(1):7781. http://dx.doi.org/10.1007/s40032-014-0101-9

Saxena, L.P., 2015. Natural and controlled symmetry breaking analyses in biological systems. Current Science, 108(5):819-824. http://dx.doi.org/10.18520/cs/v108/i5/819-824

Silverstein, D., DeCarlo, N., Slocum, M., 2007. Insourcing Innovation: How to Achieve Competitive Excellence Using TRIZ. Auerbach Publications, New York, USA.

Suh, N.P., 2004. Axiomatic Design: Advances and Application. Translated by Xie, Y.B., Yuan, X.Y., Xu, H., et al., China Machine Press, Beijing, China (in Chinese).

Todd, K.B., 2011. Tension-reducing Random Sprocket. US Patent US8066602B2.

Várkonyi, P., 2006. Emergence of Imperfect Symmetry in Engineering Optimisation and Evolution. $\mathrm{PhD}$ Thesis, Budapest University of Technology and Economics, Budapest, Hungary.

Wang, S.J., Qiu, Q.Y., Feng, P.E., 2013. A Chain Having Different Diameters Rollers. China Patent CN201220731409.7 (in Chinese).

Wang, W.Z., Wu, Z.G., Jin, W.B., 2005. A Motor Starting Method Containing Asymmetry Gap Structure. China 
Patent CN200510056169.X (in Chinese).

Xia, X.H., Xia, Y.H., 2012. Symmetry breaking during seeded growth of nanocrystals. Nano Letters, 12(11): 6038-6042.

http://dx.doi.org/10.1021/nl3040114

Yang, W.B., 1998. Structure design guidelines that satisfying thermal expansion and cold-contraction. Journal of Machine Design, (11):4-5.

http://dx.doi.org/10.13841/j.cnki.jxsj.1998.11.002

Zhou, Y.L., Yang, J.R., Gao, G., et al., 2012. An Asymmetry Automatic Gate of Elevator. China Patent CN201220153400.2 (in Chinese).

\section{中文概要}

\section{题 目：机械对称破缺的理论框架及其应用}

目 的: 为满足各种功能和性能的需求及难以避免的约 束和限制条件, 形形色色的对称破缺现象普遍 存在于各类机械系统、部件和零件之中。但目 前国内外均没有对机械对称破缺种类、作用及 其应用方法进行系统研究的报道。通过本文, 读者能系统地了解对称破缺在机械系统中的存 在和作用规律, 并能够在机械设计中科学地应
用对称破缺, 更好地实现和发挥机械的预期功 能和效益。

创新点：1. 创建机械对称破缺的概念和分类体系。2. 揭 示对称破缺与产品需求之间的关联性, 提出应 用对称破缺实现机械功能、性能和约束需求的 8 个设计原理。3. 发明一种具有对称破缺特征的 链传动结构新方案, 且其传动平稳性显著优于 现有链传动。

方 法: 1. 分析和借鉴生物学、材料结构学和建筑学等 多学科中对称破缺的研究和应用成果。2. 分析 一千多个应用对称破缺的机械实例, 并从中提 炼和总结对称破缺在机械中的效用和应用规 律。3. 将归纳出的规律应用于对称破缺创新机 械的实践。

结 论: 1. 对称破缺普遍存在于机械中, 并起着不可或 缺的作用。2. 通过对大量机械实例中对称破缺 所起作用的系统分析能够获取对称破缺作用于 机械的规律性知识。3. 应用上述规律性知识能 实现机械产品的创新。

关键词: 机械对称破缺; 概念体系; 设计原理; 设计方 法和进程; 创新实践 